Documentation et bibliothèques

DOCUMENTATION BIBLIOTHEQQUES

\title{
LOGIBASE, UN PORTRAIT BIBLIOGRAPHIQUE DU LOGICIEL QUÉBÉCOIS
}

\section{Gérard Mercure}

Volume 34, numéro 1, janvier-mars 1988

URI : https://id.erudit.org/iderudit/1052548ar

DOI : https://doi.org/10.7202/1052548ar

Aller au sommaire du numéro

\section{Éditeur(s)}

Association pour l'avancement des sciences et des techniques de la documentation (ASTED)

\section{ISSN}

0315-2340 (imprimé)

2291-8949 (numérique)

Découvrir la revue

Citer cet article

Mercure, G. (1988). LOGIBASE, UN PORTRAIT BIBLIOGRAPHIQUE DU LOGICIEL QUÉBÉCOIS. Documentation et bibliothèques, 34(1), 25-31.

https://doi.org/10.7202/1052548ar

Tous droits réservés (C) Association pour l'avancement des sciences et des techniques de la documentation (ASTED), 1988
Ce document est protégé par la loi sur le droit d'auteur. L'utilisation des services d'Érudit (y compris la reproduction) est assujettie à sa politique d'utilisation que vous pouvez consulter en ligne.

https://apropos.erudit.org/fr/usagers/politique-dutilisation/ 


\section{LOGIBASE, UN PORTRAIT BIBLIOGRAPHIQUE DU LOGICIEL QUÉBÉCOIS}

LOGIBASE', comme l'indique le nom, est une base de données de logiciels. C'est aussi un répertoire imprimé dénombrant avec son supplément de l'automne 1987 plus de 2400 logiciels conçus au Québec et les logiciels étrangers adaptés et traduits. II ne s'agit pas d'un catalogue commercial ou d'un guide de choix, mais d'un répertoire qui «vise à fournir des renseignements objectifs sur les produits disponibles» à partir des données fournies par les producteurs et distributeurs ou de l'analyse de leur documentation. $C^{\prime}$ 'est aussi, mais indirectement, un guide qui situe un logiciel par rapport à l'ensemble de la production commerciale. II s'ouvre sur le carnet d'adresses de plus de trois cents firmes productrices et auteurs québécois. Suit la section, la plus imposante de ce catalogue, des 1958 notices de la refonte du printemps 1987 et des 450 du supplément de l'automne 1987. Enfin, un jeu de six index permet l'accès au contenu tant par les marques que les titres ou les sujets.

Ce qui frappe en parcourant ce panorama du logiciel québécois, c'est la douzaine de logiciels de francisation de claviers d'écrans et d'imprimantes, le grand nombre de logiciels pédagogiques et didacticiels et le nombre plus considérable encore de logiciels consacrés à la gestion générale et aux applications professionnelles. Ce répertoire donne rapidement un aperçu des logiciels disponibles dans un domaine donné, que ce soit pour bibliothèques et centres de documentation ou pour bureaux d'avocats, par exemple.

Ces logiciels sont en effet regroupées par grands centres d'intérêt: logiciels de base et utilitaires, logiciels éducatifs, didacticiels, logiciels professionnels d'application générale, logiciels professionnels spécialisés et logiciels familiaux et de divertissement. Le cadre de classement de LOGIBASE a été développé par la Centrale des bibliothèques $^{2}$ et pour la classe des logiciels professionels à partir de la Classification des activités professionnelles du Québec. Plusieurs index, aux auteurs et producteurs, aux titres, aux domaines d'application, à la marque des ordina- teurs et aux systèmes d'exploitation ainsi qu'aux fournisseurs permettent le repérage de l'information. Chaque index est imprimé sur un papier de couleur différente pour bien le distinguer de l'index voisin et rendre ainsi plus rapide la consultation de cet ouvrage de référence.

Une notice-type illustre en tout début du répertoire les éléments décrivant un logiciel: d'abord un numéro d'ordre auquel renvoient les index en fin de volume. Lorsqu'un logiciel se présente en modules distincts, une notice est rédigée pour chacun de ces parties autonomes, en plus de la notice principale décrivant le logiciel dans son ensemble. Un indicateur de conception québécoise (Q) distingue les logiciels québécois des logiciels traduits qui, par défaut, ne portent aucune mention. L'indicateur de la langue d'affichage, française ou anglaise ou les deux, dissipe tout doute. II s'agit là, soit dit en passant, d'un élément de description que l'on souhaiterait bien retrouver dans les revues de micro- informatique et les catalogues de fournisseurs. II n'est pas toujours évident, même dans les revues françaises, que le titre soit représentatif de la langue. La mention de traduction est rarement indiquée. On observe même une complaisance chez les Francais pour des titres à l'anglaise et chez les Américains pour des titres à la française. Si Symphony traduit en français demeure Symphony, Déjà!, naturalisé américain, affiche ses origines françaises avec ses deux accents et son point d'exclamation tandis que 4 e Dimension est devenu en traversant l'Atlantique 4th Dimension... Dans le cas de Best-Seller, inscrit au répertoire des logiciels québécois, on peut se demander s'il s'agit d'un néologisme ou d'une traduction. LOGIBASE, par son indicateur confirme l'origine québécoise ainsi que la langue d'affichage, l'anglais dans ce cas. La notice descriptive précise: «Documentation disponible en anglais: rapports générés dans huit langues» ( $p$. 299). Ce cas illustre que la mention de la langue n'est pas une réalité toujours simple à décrire pour dissiper toute ambiguité quant à la langue d'affichage, à la langue de la documentation et à la langue de production des états imprimés.

1 LOGIBASE; répertoire des logiciels québécois Printemps 1987. Montréal, Centrale des bibliothèques, 1987468 p. - Supplément. (Automne 1987). 107 p

2. La Centrale des bibliothèques a changé son nom et s'appelle maintenant Services documentaires multimedia Inc 


\section{NOTICE EXPLIQUÉE}

Numéro d'ordre dans la publication (pour le repérage dans les index)

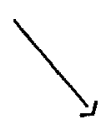

0040
Indicateur de

conception québécoise

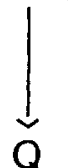

\section{F A}

Titre

Mac Keymeleon / François Morel et Pierre

Pilon. -- Québec : Logiciel Avenue, c1985.

1 disquette ; $9 \mathrm{~cm}+$ Livret d'instructions.

Pour ordinateurs Apple Macintosh, 128K, MAC OS $59,95 \$[1987]$ Équipement

Accès sujets: *Clavier français. ${ }^{*}$ Clavier scientifique. *Imprimantes - Caractères français. *Imprimantes - Caractères scientifiques. ${ }^{*}$ Générateurs de caractères trançais. ${ }^{\star}$ Générateurs de caractères scientifiques.

Code de classement

AF/M113

Ce logiciel permet d'adapter au Macintosh une configuration de clavier standard (QWERTY, DVORAK, AZERTY), de concevoir de nouvelles dispositions de clavier et de passer, en tout temps, d'une configuration de clavier à une autre. II est possible de définir des touches mortes et de créer des combinaisons de doubles clés. II comprend également des jeux de caractères originaux incluant toutes les majuscules françaises accentuées et plusieurs caractères spéciaux (boîte pour formulaire, exposants, elc.). Le logiciel facilite aussi l'utilisation de jeux de caractères spéciaux (grecs, cyrilliques, mathématiques, scientifiques, etc.). Les menus et explications sont disponibles en français, en anglais ou en espagnol. Enfin, Mac Keymeleon est compatible avec tous les logiciels Macintosh standard.
Analyse

de contenu

Références

Pour en apprendre davantage: * $L$ 'Apôtre, No 32, 8

bibliographiques nov. 1985. * Macworld, sept. 1986. ${ }^{\star} \mathrm{C}$.J. Weigand, MACazine, avril 1986, p. 82.

\section{Source: AVENUE}

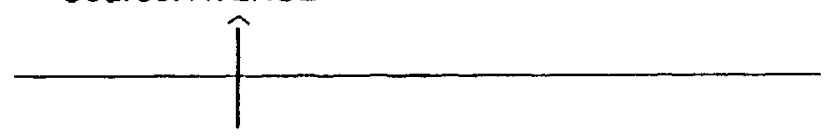

Code d'identification du distributeur 


\section{Les éléments de la notice}

L'entrée principale au titre est complétée par la mention des auteurs, de la version, du producteur et de la date de parution. Suivent les caractéristiques de l'équipement requis, du système d'exploitation utilisé et du prix. Viennent ensuite les termes et expressions permettant l'accès aux sujets. La présence de ces vedettes-matière dans la notice permet de situer rapidement le logiciel à l'intérieur de la catégorie à laquelle il appartient. Suivent les codes de classement et le numéro de la notice qui selon la terminologie des bibliothèques s'appelleraient respectivement cote et numéro d'accession. Autre indication utile pour situer le logiciel par rapport à sa catégorie d'utilisateurs: I'indice de public cible et de valeur/ utilité, allant des enfants de maternelle et d'âge préscolaire aux professionnels ou enseignants en passant par les adultes renseignés et le public spécialisé. La Centrale n'applique l'indice d'utilité qu'aux logiciels éducatifs et aux jeux, et de moins en moins, dans ses notices les plus récentes. Le grand nombre de logiciels associés à la lettre $P$ pour professionnels ou enseignants appliquée à la majorité des logiciels du Supplément (Automne 1987) montre dans quel sens évolue le marché du logiciel au Québec.

\section{L'analyse descriptive}

La Centrale dit bien dans la préface qu'elle «n'évalue aucun logiciel». Elle signale cependant les appréciations faites par d'autres organismes et les publications spécialisées. Elle fournit les références bibliographiques d'évaluations faites dans les revues tant québécoises qu'américaines, s'il s'agit d'une traduction, sous la rubrique «Pour en apprendre davantage». Avec les années, le nombre de ces références pour un logiciel donné a diminué. Serait-ce imputable au fait que les revues font de moins en moins de critiques de logiciels à la pièce, procédant dans des analyses comparatives par familles en raison du grand nombre de produits nouveaux qu'il faudrait autrement analyser un par un? Lorsqu'elles sont signalées, les références apparaissent en fin de notice à la suite d'une description du logiciel qui en résume les caractéristiques. Ce commentaire, dont la source est ordinairement le producteur, est rédigé dans un style suivi, uniformisé mais non monotone, la formule variant légèrement d'une notice à l'autre. Elle évite les qualificatifs qui pourraient avoir la couleur d'une appréciation favorable ou défavorable. Les plus anciennes notices sur les didacticiels rendaient compte de
I'appréciation de I'INRS-Education ou des revues de micro-informatique. Avec la parution du Supplément (Eté 1986), tous les logiciels sont traités de la même façon et le résumé descriptif tend à s'abréger à une dizaine de lignes ou moins. Pour les logiciels d'affaires, il ne faut pas confondre ces références avec la mention en fin de notice de l'origine du commentaire, qu'est la revue Informatique et bureautique. Cette revue, qui s'adresse au monde des affaires, participe au dépistage des logiciels et publie depuis 1985, en août de chaque année, un numéro sur les logiciels développés pour les besoins de l'entreprise. Son Répertoire des logiciels d'affaires fabriqués au Québec ${ }^{3}$ est réalisé à partir de ces données. Grâce à une entente avec Informatique et bureautique, la Centrale reprend ces notices, à quelques détails stylistiques près, pour les intégrer à sa base.

\section{Les index}

La diversité des index distingue LOGIBASE d'un répertoire commercial dont les approches sont réduites à des classements alphabétiques sous de grandes catégories. On perçoit ici le savoirfaire de la Centrale dans la confection d'un répertoire bibliographique. Ainsi, l'index des noms est un véritable catalogue d'autorité établissant le nom complet de l'entreprise, suivi entre parenthèses du nom de la ville et de la province d'origine. Pour une recherche rapide, cela peut être gênant car il faut revenir à la Liste des fournisseurs du début pour savoir, par exemple, que la firme VAUDREUIL sera indexée sous "Systèmes d'information Vaudreuil (Vaudreuil, Québec)». Mais, comme on le constate ici, un tel traitement évite de confondre le nom de l'entreprise et son lieu d'origine. Le crédit est donné à l'auteur intellectuel, lorsqu'il est identifiable, ce qui n'est pas la pratique de l'anonymat corporatif des catalogues commerciaux. Sous ce nom, apparaissent les titres des logiciels et leurs codes de repérage dans la base, ce qui permet de parcourir d'un seul coup d'oeil la production d'une firme ou d'un auteur. L'index des titres présente le problème du regroupement des modules d'un même logiciel. L'astuce réside dans la façon de traiter le module comme un soustitre. Ainsi le module des acquisitions et de Catalogage et repérage de Turbo-biblio sont ainsi classés: «Turbo-biblio Acquisitions. 1660», suivi de «Turbo-biblio Catalogage et repérage. 1661».

L'index des séries qui équivaut en terme bibliographique à l'index des collections représente à peine une page, cette pratique d'un

3. «Répertoire des logiciels d'affaires fabriqués au Québec». Informatique et bureautique, vol. 6 , no 6 (juillet/août 1985), $54 \mathrm{p}$. 
regroupement thématique n'ayant cours que pour les logiciels éducatifs. L'index des sujets reprend les éléments de la rubrique Accès sujets de la notice et vient compléter l'approche thématique de la table des matières. Malheureusement, le traitement par vedettes-matière, bien maîtrisé par les bibliothécaires, n'est pas très convivial pour le profane. Si «Education préscolaire» a des allures de langage naturel, «Electriciens, Bureau d' - Contrats - Gestion» rappelle la recherche au sujet dans les bons vieux catalogues sur fiches. La Centrale entend assouplir cet index des sujets à l'occasion d'une prochaine refonte en ajoutant un réseau de renvois. Malgré cette difficulté d'accès que représentent le recours aux vedettesmatière, cet index est riche en rapprochements et constitue en soi une source thématique de renseignements. Ainsi, tous les titres relatifs à l'enseignement de l'arithmétique se retrouvent regroupés, tout comme les logiciels de gestion de bibliothèque contenant un module de traitement des périodiques sont rassemblés sous une même vedette.

L'index des marques rassemble respectivement sous les marques d'ordinateurs et de systèmes d'exploitation les logiciels décrits dans LOGIBASE. Si la grande diversité des marques est frappante, le grand nombre des systèmes d'exploitation l'est davantage. La majorité des logiciels se retrouvent sous les marques Apple, Macintosh et IBM-PC et leurs systèmes respectifs d'exploitation. Une seule liste est fournie pour PC-DOS et MS-DOS, les deux systèmes offrant les mêmes produits. On est loin de la normalisation cependant. Dans le seul secteur des bibliothèques et des centres de documentation, une douzaine de systèmes d'exploitation différents sont utilisés par les créateurs de logiciels pour petits et grands ordinateurs.

Le dernier index, l'index des fournisseurs, classe les logiciels par producteurs fournissant ainsi des indications relatives au domaine d'activité de la maison: SIETI avec sa série de programmes PROMEDIC en médecine, SINED EITROL avec ses logiciels éducatifs, etc.

\section{L'exhaustivité}

Constituer ainsi un répertoire des logiciels québécois constitue en soi un exploit en raison même de la diversité des fournisseurs. plus de 300 , et du fichier des 2000 produits recensés qu'il faut tenir à jour. Le projet fit appel au départ à la participation de la revue Informatique et bureautique, du Laboratoire d'évaluation de logiciels et de didacticiels de l'INRS-Education, des ministères de l'Education et de l'Industrie, du Commerce et du Tourisme ainsi que d'une boutique maintenant disparue, IPL Informatique de Montréal. Née du besoin de recenser les logiciels éducatifs, LOGIBASE a vite élargi ses horizons à l'ensemble des logiciels québécois, quels que soient le type d'application et la taille de l'ordinateur.

L'index des noms qui suit la section des notices est révélatrice d'un marché aujourd'hui très fragmenté, chaque maison ayant de deux ou trois à 50 titres à son catalogue. Producteur et distributeur ne font qu'un dans bien des cas car très peu de producteurs confient à une autre entreprise la distribution de leurs logiciels, comme le fait DIL (Didacticiel international et laboratoire) avec l'éditeur Guérin. Selon cet index, la DGES (Direction de l'enseignement collégial) serait le plus gros distributeur de logiciels de ses collèges et pas de tous - avec quelque 75 titres à son catalogue.

Dans son numéro spécial de janvier 1988 intitulé "Trouvez vos fournisseurs" ${ }^{4}$, Informatique et bureautique établit la liste des fabricants et distributeurs. En faisant un recoupement selon le statut de fabricant et distrubuteur, on constate qu'il y a deux fois plus de fabricants et de distributeurs que de simples distributeurs. Pour établir un portrait plus exact de la situation, il faudrait aussi distinguer dans le décompte les filiales québécoises de grands distributeurs canadiens et étrangers, qui ont pour noms Softsel Canada, Frantek Computer, Compuserv ou Ollisoft. Les fabricants de logiciels ont jusqu'à ce jour préféré assurer eux-mêmes la diffusion, l'implantation de leurs produits de même que l'assistance technique plutôt que d'en confier la promotion à un distributeur. Une tendance nouvelle s'affirme, compte tenu de la vie relativement courte des produits informatiques et d'une clientèle souvent spécialisée mais aussi dispersée, de s'associer à un groupe informatique tel que Logidisque ou Logibec. Pour constituer et tenir à jour une liste des fabricants et de leurs produits, il faut donc que la Centrale les identifient et les relancent un à un. Cela constitue en soi une entreprise méritoire et un maintien en état d'alerte constant.

LOGIBASE est-il un répertoire exhaustif? Pour les mêmes raisons, il est aussi difficile de vérifier. L'écho des salons comme celui de la fabrication 
automatisée dans Le Devoir du 18 février $1988^{5}$, nous a permis de "valider» la présence de deux logiciels, SIFIA de Jovaco Inc. destiné aux architectes et aux ingénieurs et Le Grammairien de Technogram à l'intention de l'entreprise, distribué par Logidisque, deux logiciels dont on faisait la mention dans I'article. SIFIA n'apparaît pas dans les pages de LOGIBASE, mais après confirmation auprès de Jovaco, il s'agit d'une version adaptée d'un de ses logiciels, Contrôle des coûts de projets à une clientèle particulière parue après le Supplément (Automne 1987). Dans le cas du Grammairien, mention est faite de ce logiciel dans LOGIBASE, mais non de son nouveau distributeur, Logidisque qui depuis peu assure la diffusion pour Technogram. Compte tenu du décalage entre la date de publication de LOGIBASE et de celle du Salon de la fabrication automatisée, l'information de LOGIBASE était, à I'heure de tombée, exacte et complète.

Une autre façon de procéder consiste à prendre un secteur dont on connait la production, comme les bases de données documentaires par exemple, et de vérifier la présence des logiciels en excluant les tous récents cependant. Dans un numéro spécial de Informateur-Logiciel ${ }^{6}$ sur l'informatique documentaire, un profil de 17 entreprises est proposé aux lecteurs. Deux de ces firmes n'apparaissent pas au fichier des fournisseurs de LOGIBASE, il s'agit de Trellissys Software Corp. et de la société de programmantion COBA Inc. En nous enquérant auprès de ces maisons, nous avons constaté que La Centrale nous avait devancé dans la démarche pour s'assurer qu'elles seraient présentes ainsi que leurs produits dans la prochaine refonte du printemps 1988.

II en échappe sans doute quelques-uns à la vigilance de la Centrale, soit que l'auteur d'un logiciel ne connaisse pas LOGIBASE ou que la Centrale ne puisse pas même soupçonner l'existence du nouveau logiciel. C'est probablement le cas de BBI/PC (Banque Bibliographique interactive) d'Indexatech ${ }^{7}$, oeuvre patiente d'un informaticien de Hull. Dans un autre cas, le silence de LOGIBASE peut s'expliquer par le caractère non commercial de l'organisme qui a présidé à la recherche et au développement d'un logiciel. Comment expliquer autrement l'absence des produits tels que SATO (Système d'analyse de texte par ordinateur) et Déredex-EXPERT, un générateur de système expert, tous deux développés par le Centre d'ATO (Analyse de texte par ordinateur) de l'Université du Québec à Montréal? Plus la Centrale et LOGIBASE seront connus et accrédités auprès du milieu, plus l'inventaire sera facile à tenir à jour. Un jugement aussi favorable que celui de Gérard Blanc de Micro-Gazette ${ }^{8}$ contribuera à renforcer la position de LOGIBASE et l'intérêt des producteurs de logiciels à s'y inscrire: «L'information contenue dans LOGIBASE nous apparaît comme une source extrêmement fiable et d'une remarquable qualité technique».

\section{LOGIBASE et les autres}

La Centrale a dû innover en matière de catalogage. Le format de LOGIBASE a été éléboré de 1982 à 1985 et sa première édition est parue en 1985. C'est dire qu'il a fallu décider du format bibliograhique et du mode d'indexation avant que ne soient publiées les Guidelines for using AACR2 chapter 9 for cataloging microcomputer software ${ }^{9}$ parus en 1984 et les Guidelines on subject access to microcomputer software de I'ALA ${ }^{10}$, en 1986. Ces propositions sont encore les bases de la pratique actuelle du catalogage des logiciels dans les bibliothèques. Elles suppléent aux règles des RCAA, chapitre 9 qui ont été conçues en fonction des programmes de l'informatique lourde des années 1970, accessible par terminaux et non pour la description d'articles plus près du matériel audiovisuel que sont les progiciels de la microinformatique actuelle. Même si la Centrale avait pu régler sa pratique en référant à un code mieux adapté, il lui aurait fallu de toute façon modifier les règles relatives à la description de l'article puisqu'il ne s'agit pas dans ce type de répertoire d'une description physique d'un coffret que I'on tient en main, mais du signalement d'un ouvrage disponible chez un fournisseur Le Centrale a dû également résoudre des problèmes liés à une terminologie de l'informatique en pleine évolution. II lui aura fallu choisir entre "tableur» et "chiffrier électronique», inventer le mot «partagiciel» pour "shareware» et accepter le verbe «émuler», cher aux informaticiens. Dans le cas de termes plus techniques comme «anté-
5. Pierre Deschamps, "Les ombres de Big Brother et d'Oncle Picsou planent sur le salon de la fabrication automatisée", Le Devoir, jeudi 18 février 1988. p. 6.7

6. «Profils d'entreprises», Informateur-Logiciel; le magazine de l'informatique pour le gestionnaire, vol. 2, no 9 (1988), 18 . 23

7 Trop récent pour être décrit dans le Supplément (Automne 1987), ce logiciel, mis sur le marché en juillet 1987 n'apparaissait pas encore dans la base interrogeable en ligne en mars 1988
8. Gérard Blanc, "LOGIBASE: dis-moi ce que tu cherches et je te dirai où le trouver", Micro-gazette (Janvier 1987), 57

9. American Library Association. Committee on Cataloging: Description and Access. Guidelines for using AACR2 chapter 9 for cataloging microcomputer software Chicago. ALA, 1984. 32 p

10. Guidelines on subject access to microcomputer software, Chicago, ALA, 1986. 27 p. 
mémoire» (et non anti-mémoire, p. 152, dans une notice de 1985), elle a fait suivre du terme anglais plus connu qu'est «disk-caching». Cette mise au point du vocabulaire technique a été réalisée avec la collaboration de l'Office de la langue française.

D'autres guides imprimés ont été publiés au cours des mêmes années comme sous-produits également de bases de données accessibles en ligne. Ces répertoires sont de portée soit internationale comme The Software Catalog: Microcomputers, $1983^{11}$ et The Software Catalog: Minicomputers, $1983^{12}$ de Elsevier, soit nationale comme Software Encyclopedia, $1986^{13}$ de Bowker des logiciels disponibles aux Etats-Unis. Ils ont toutefois une facture davantage inspirée des catalogues commerciaux que bibliographiques. Ainsi Elsevier identifie les logiciels par leurs fournisseurs et Bowker les regroupe dans l'ordre des titres, les deux dans une présentation différente de l'ordre et des éléments d'une fiche bibliographique.

Au Canada, Maclean Hunter publie depuis 1982 le Canadian Directory of Software. C'est un répertoire des logiciels disponibles commercialement au pays. Comme source complémentaire d'information, le Software User Evaluation survey, résultat d'un sondage annuel, et publié par le même éditeur. II existe pour les didacticiels la base de données sur les logiciels éducatifs du Conseil des ministres de l'Éducation (Canada) ${ }^{14}$. Celle-ci est conçue dans le but de signaler et d'évaluer ces logiciels selon de nombreux critères standardisés avec cote graduée d'appréciation allant de «1: $V u$ et rejeté» à «4: Évalué et recommandé». Accessible en ligne, cette base fait l'objet de catalogues imprimés thématiques tel celui sur la cognition qui contient une soixantaine de logiciels.

Dans le monde francophone, paraît depuis 1987 , le Répertoire des Logiciels produits et commercialisés à Bruxelles et en Région wallonne ${ }^{15}$ dont la présentation s'inspire largement de LOGIBASE. C'est, paraît-il, une retombée indirecte du Sommet francophone de Québec de 1987. Ce répertoire décrit brièvement environ 700 logiciels belges et comprend un seul index, celui des descripteurs. On ne peut que souhaiter que la France, grande productrice de logiciels, dresse également son inventaire national. Quant au Québec, maintenant qu'il a proposé avec LOGIBASE une "norme pratique» de réalisation aux pays de langue française, ne devrait-il pas s'attaquer à l'évaluation de ses logiciels en publiant une revue spécialisée sur le sujet? C'est là un rêve peut-être. Car à raison d'environ 500 logiciels nouveaux par années, la mise à jour de LOGIBASE constitue déjà un travail imposant qui risque de s'allourdir avec les années.

L'information dans LOGIBASE est organisée de façon rationnelle. Les index fournissent les éléments suffisants pour une consultation aisée. La redondance est réduite au minimum. Même la Table des matières est mise à profit car elle suggère les grandes lignes du cadre de classification. Ainsi, on retrouvera à partir de «GB Gestion de fichiers et de bases de données» de la Table des matières les notices regroupées sous la cote GB et portant sur les fichiers d'adresses, les gestionnaires généraux de fichiers et de bases de données.

Certains souhaiteraient un commentaire plus critique. En se situant entre le répertoire commercial aux données bibliographiques succinctes et aux index moins élaborés qu'est le répertoire annuel d'Informatique et bureautique et le guide de choix avec des critères détaillés d'évaluation qu'est la Base de données du CMEC, la Centrale a adopté une position réaliste. Elle mise sur une information disponible et vérifiable, et un format suffisamment simple pour ne pas occasionner de retard dans la mise à jour Elle peut ainsi maintenir un coût de production abordable. A ce titre, le prix de la refonte, de l'ordre de 75,00 \$ et de la mise à jour, d'environ 25,00 $\$$ sont tout à fait raisonnables pour ce genre d'ouvrage.

\section{Intérêt pour les bibliothécaires}

Pour le spécialiste de la documentation, deux sections de LOGIBASE seront particulièrement utiles: la sous-section «GB: Gestion de fichiers

11. Software Catalog: Microcomputers, 1987, Elsevier, 1987 $3 \mathrm{v}$.

12. Software Catalog: Minicomputers, 1986, New York, N.Y., Elsevier, 1986. 1050 p. (Science Publishing)

13. The Software encyclopedia 1987/1988, New York, R.R. Bowker, 1987. 2 v.

14. Conseil des ministres de l'Education (Canada). Cognition, base de données du CMEC sur les logiciels éducatifs catalogue de logiciels. Toronto, 1986. $54 \mathrm{p}$.

15. Répertoire des logiciels produits et commercialisés à Bruxelles et en Région wallonne. Bruxelles, Région wallonne, 1987 
et de bases de données» et la sous-section «PO: Organismes d'enseignement» sous Bibliothèques et centres de documentation. En regroupant ce qui est bibliothèque et documentation sous la bannière de l'enseignement, LOGIBASE est fidèle à ses origines éducatives. Avec les 39 logiciels ou modules de gestion de bibliothèques et de centres de documentation, le secteur de l'infor matique documentaire est florissant. II serait peut-être temps d'en faire un secteur autonome d'activité au même titre que la Construction et les Transports qui possèdent leur propre rubrique. La revue Informatique et bureautique dans son numéro spécial annuel classe plutôt ces logiciels dans la famille des centres de documentation, ce qui est plus juste mais également discutable. Audelà de l'argument du nombre, il y a celui de la logique: la gestion documentaire automatisée ne s'applique pas seulement aux organismes d'enseignement, mais de plus en plus, par exemple au secteur de la santé. Mais, cela soulève le problème classique de tout cadre de classification, celui de satisfaire tout le monde et son père, le plan d'ensemble et la vision du spécialiste. Un tel cadre de classification est déjà un compromis entre une organisation par genres de logiciels et un regroupement par domaines d'applications.

LOGIBASE est un ouvrage de référence indispensable à toute bibliothèque ou centre de documen- tation à moins de préférer la version en ligne. LOGIBASE, comme base de données informatisée, est accessible en accès direct en communicant directement avec l'ordinateur de la Centrale ou par le réseau iNet et le serveur ISTInformathèque au Canada et en France par Minitel via le SUNIST (Serveur universitaire pour l'information scientifique et technique). Pour le bibliothécaire qui veut automatiser son service, la consultation de ce catalogue des logiciels québécois constitue la première étape de son "magasinage» auprès des producteurs de logiciels documentaires. Dans le milieu plus large des affaires et de l'éducation, ce répertoire constitue une sorte d'annuaire pour un premier contact de l'utilisateur avec le producteur de logiciel, que ce soit pour des fins de gestion ou d'enseignement.

Logibase est, de par sa facture et son envergure, une bibliographie nationale du logiciel québécois; ses refontes et mises à jour successives lui confèrent aussi un rôle analogue à celui d'une liste des Livres disponibles. Car ce répertoire réflète à la fois l'image de l'édition informatique et l'état du marché du logiciel au Québec.

\section{Gérard Mercure}

École de bibliothéconomie et des sciences de l'information Université de Montréal

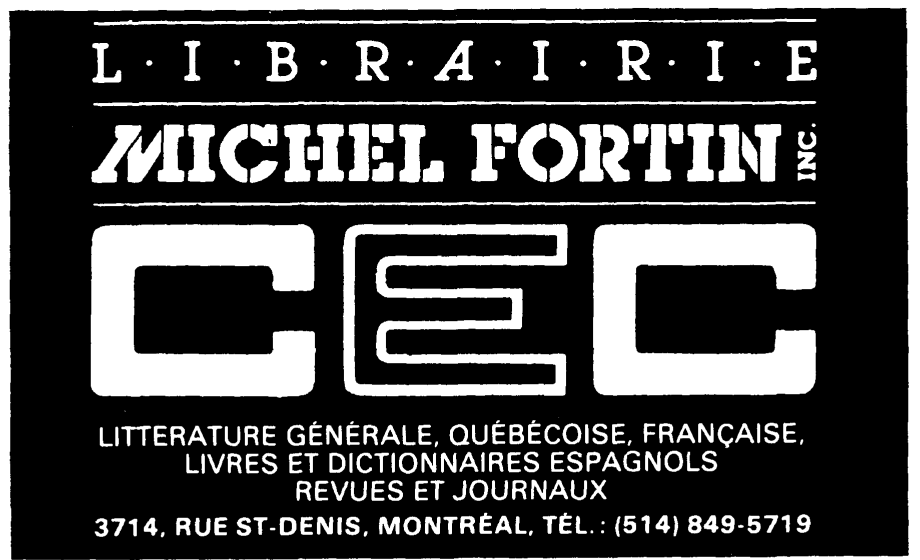

